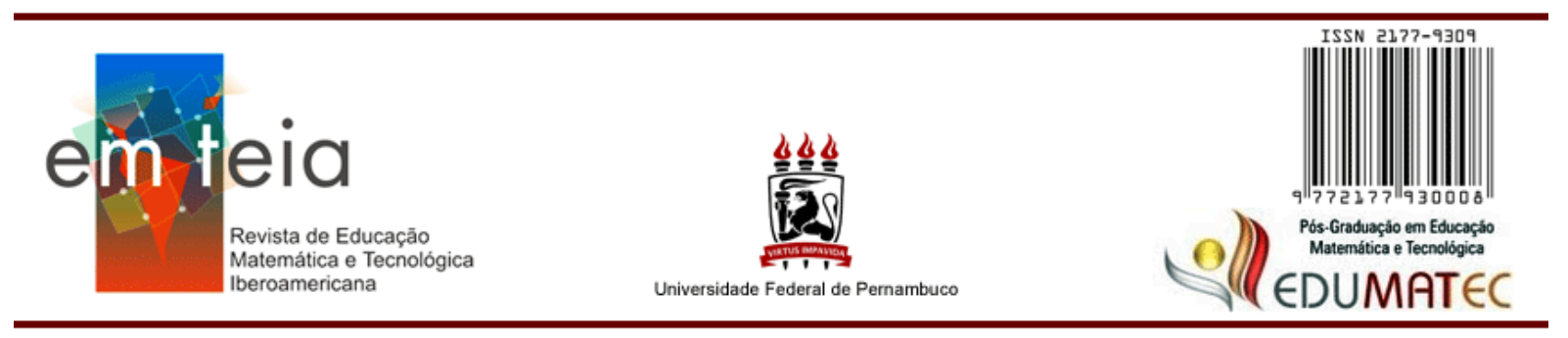

\title{
A FUNÇÃO EXPONENCIAL NA PESQUISA EM EDUCAÇÃO MATEMÁTICA: como dialoga com a BNCC?
}

\section{THE EXPONENTIAL FUNCTION IN RESEARCH IN MATHEMATICAL EDUCATION: how do it dialogue with BNCC?}

\author{
Gleidson Santos Correia \\ Especialista em Metodologia do ensino da Matemática \\ Centro Territorial de Educação Profissional Litoral Sul - Bahia - Brasil \\ gleidson.correia@enova.educacao.ba.gov.br \\ https://orcid.org/0000-0003-1011-7520 \\ Irene Mauricio Cazorla \\ Pós-doutora em Educação Matemática \\ Universidade Estadual de Santa Cruz - Bahia - Brasil \\ icazorla@uol.com \\ https://orcid.org/0000-0003-3028-5513
}

\begin{abstract}
Resumo
De acordo com a Base Nacional Comum Curricular (BNCC), a função exponencial é um objeto que faz parte do currículo de Matemática do Ensino Médio que elenca competências que os estudantes devem desenvolver em Matemática, bem como habilidades específicas no estudo da função exponencial. O objetivo desta pesquisa é realizar uma revisão sistemática de teses e dissertações nos programas de PósGraduação em Ensino de Ciências e Matemática ou Educação Matemática, envolvendo a função exponencial na Educação Básica, tendo como parâmetros as competências e habilidades propostas pela BNCC. A busca foi realizada no Banco de Teses e Dissertações da Capes, sendo o universo de análise composto por 50 trabalhos que foram submetidos à Análise de Conteúdo para verificar como essas produções dialogam com as categorias criadas a partir da BNCC. Verifica-se que, embora a maioria das pesquisas não faça menção à BNCC, essas conseguem estabelecer diálogos em diversos quesitos, como Resolução de Problemas e Modelagem Matemática, utilização de diversos registros de representação, uso de tecnologias e relação com a Matemática Financeira e a Biologia. Apesar de alguns trabalhos abordarem a contextualização e a interdisciplinaridade, percebe-se a necessidade de contextos significativos, do papel mais ativo do estudante e do desenvolvimento de habilidades de comunicação no processo de aprendizagem.
\end{abstract}

Palavras-chave: Função exponencial, Ensino Médio, BNCC, revisão sistemática, pós-graduação.

\begin{abstract}
According to the National Common Curricular Base (BNCC), the exponential function is an object that is part of the High School Mathematics curriculum that lists skills that students must develop in Mathematics, as well as specific skills of the exponential function study. The objective of this research is to carry out a systematic review of master and doctoral theses in the Graduate Programs in Science
\end{abstract}


and Mathematics Education or Mathematical Education involving the exponential function in Basic Education, taking as parameters the competences and skills proposed by the BNCC. The search was carried out at the Bank of Theses from Capes (Coordination of the Improvement of Higher Education Personnel), and the universe of analysis consisted of 50 theses, which were submitted to Content Analysis to verify how these productions dialogue with the categories created from the BNCC. We can verify that, although most research does not mention BNCC, they manage to establish dialogues on several issues, such as Problem Solving and Mathematical Modeling, use of various representation records, use of technologies and relationship with Financial Mathematics and Biology. Although some theses address contextualization and interdisciplinarity, there is a need for significant contexts for the student's more active role and the development of communication skills in the learning process.

Keywords: Exponential function, High School, BNCC, systematic review, post graduation.

\section{Introdução}

As funções matemáticas são objetos valiosos para a modelagem de diversos fenômenos que permeiam a natureza e a vida humana. Dentre elas, destaca-se a função exponencial (FE), a qual modela satisfatoriamente os juros compostos, o processo de reprodução celular, o contágio de algumas doenças infecciosas e o crescimento de algumas populações, por exemplo. No Brasil, a FE é estudada na Educação Básica (EB), na etapa do Ensino Médio (EM), no componente curricular de Matemática.

Por essa razão, a FE tem sido investigada em diversas pesquisas. Silva e Panossian (2017) e Carreta e Godoy (2018) investigaram como ela é tratada nos principais livros de Matemática do EM, enquanto Breunig e Gabbi (2011), Oliveira e Calejon (2016) e Silva e Costa (2020) apresentaram propostas de sequências didáticas para o seu ensino. Já Bonotto e Bisognin (2015), Mendonça e Pires (2016), Eisermann et al. (2017), Lorencini, Nogueira e Rezende (2018) e Cardozo e Possamai (2019a, 2019b) coletaram dados empíricos com estudantes do EM, verificando o ensino da FE sob diversas perspectivas.

Recentemente, foi aprovada a Base Nacional Comum Curricular - BNCC (BRASIL, 2018), que normatiza a organização do currículo da EB. Este documento elenca competências gerais que devem ser desenvolvidas ao longo deste nível educacional, bem como competências específicas, objetos de conhecimento e habilidades para os diversos componentes curriculares. A FE está inserida no EM, podendo ocorrer da $1^{\mathrm{a}}$ a $3^{\mathrm{a}}$ série, pretendendo desenvolver várias habilidades no seu estudo.

Obviamente, as pesquisas envolvendo a FE realizadas até 2018 não consideraram essas competências e habilidades, pois o documento ainda não havia sido publicado e, mesmo as pesquisas realizadas em 2019 e 2020 ainda estariam em descompasso, uma vez que as redes de ensino estavam adequando seus currículos à nova normativa.

Todavia, isso não impede que tais pesquisas possam ser analisadas a partir das competências e habilidades preconizadas pela $\mathrm{BNCC}$, pois isso permitirá verificar quais 
aspectos já foram abordados e quais as lacunas existentes, norteando novas linhas de investigação. Diante disso, o objetivo desta pesquisa, que faz parte de uma investigação maior em nível de mestrado em desenvolvimento em uma universidade pública do Estado da Bahia, foi o de realizar uma revisão sistemática da produção científica, teses e dissertações, nos programas de Pós-Graduação brasileiros em Ensino de Ciências e Matemática e em Educação Matemática, envolvendo o ensino e aprendizagem da FE na EB e tendo como foco de análise as competências e habilidades propostas pela BNCC.

\section{A função exponencial e o seu ensino na Educação Básica}

Espindola (2016), Silva e Panossian (2017) e Carreta e Godoy (2018) observaram que geralmente, nas aulas de EM e no livro didático, a introdução à FE é realizada por meio de sua definição e características algébricas, conforme apresentados nos livros de Lima (2013) e Dante (2013), sem nenhuma contextualização e, por vezes, tendo o desenvolvimento das aulas pautado em resolução de exercícios contidos nos livros didáticos.

Embora a caracterização e o rigor matemático envolvido nas definições sejam importantes, isso não é suficiente, pois, conforme a BNCC, ao estudar esta função os estudantes devem desenvolver diversas habilidades, como as listadas no Quadro 1.

Quadro 1 - Habilidades propostas pela BNCC para a aprendizagem da função exponencial

\begin{tabular}{|l|l|}
\hline \multicolumn{1}{|c|}{ Código } & \multicolumn{1}{c|}{ Descrição da habilidade } \\
\hline EM13MAT203 & $\begin{array}{l}\text { Planejar e executar ações envolvendo a criação e a utilização de aplicativos, jogos (digitais } \\
\text { ou não), planilhas para o controle de orçamento familiar, simuladores de cálculos de juros } \\
\text { compostos, dentre outros, para aplicar conceitos matemáticos e tomar decisões. }\end{array}$ \\
\hline EM13MAT303 & $\begin{array}{l}\text { Resolver e elaborar problemas envolvendo porcentagens em diversos contextos e sobre juros } \\
\text { compostos, destacando o crescimento exponencial. }\end{array}$ \\
\hline EM13MAT304 & $\begin{array}{l}\text { Resolver e elaborar problemas com funções exponenciais nos quais é necessário } \\
\text { compreender e interpretar a variação das grandezas envolvidas, em contextos como o da } \\
\text { Matemática Financeira e do crescimento de seres vivos microscópicos, entre outros. }\end{array}$ \\
\hline EM13MAT403 & $\begin{array}{l}\text { Comparar e analisar as representações, em plano cartesiano, das funções exponencial e } \\
\text { logarítmica para identificar as características fundamentais (domínio, imagem, crescimento) } \\
\text { de cada uma, com ou sem apoio de tecnologias digitais, estabelecendo relações entre elas. }\end{array}$ \\
\hline EM13MAT404 & $\begin{array}{l}\text { Analisar funções definidas por uma ou mais sentenças (tabela do Imposto de Renda, contas } \\
\text { de luz, água, gás etc.), em suas representações algébrica e gráfica, identificando domínios de } \\
\text { validade, imagem, crescimento e decrescimento, e convertendo essas representações de uma } \\
\text { para outra, com ou sem apoio de tecnologias digitais. }\end{array}$ \\
\hline EM13MAT508 & $\begin{array}{l}\text { Identificar e associar sequências numéricas (PG) a funções exponenciais de domínios } \\
\text { discretos para análise de propriedades, incluindo dedução de algumas fórmulas e resolução } \\
\text { de problemas. }\end{array}$ \\
\hline
\end{tabular}

Fonte: Elaborado pelos autores com base em BRASIL (2018)

Observa-se que o processo de aprendizagem dos estudantes, na perspectiva da BNCC, deve ser ativo, reflexivo e crítico, indo muito além da memorização de regras algébricas ou definições. Isso pode ser identificado pelos verbos utilizados na descrição das habilidades que 
devem ser desenvolvidas, tais como planejar, elaborar, executar, identificar, comparar etc. Fica evidente que o ensino deve permitir e incentivar os estudantes a construírem ativamente os conhecimentos, compreendendo a FE em suas diversas representação.

As habilidades listadas no Quadro 1 revelam também que, ao estudar a FE, é necessário relacionar este objeto matemático a outros conhecimentos da própria Matemática, como os juros compostos, as progressões geométricas e a Estatística. Além disso, é notória a necessidade de relacioná-la também às outras Ciências, como as Ciências Sociais e as Ciências da Natureza, revelando assim uma perspectiva interdisciplinar. Outro destaque percebido refere-se à possibilidade e incentivo ao uso de tecnologias, o que pode ser visto em quatro das habilidades listadas. A utilização de diversos registros de representação, como a língua materna, o registro algébrico e o gráfico também recebem destaque nessas habilidades.

Percebe-se, ainda, uma ênfase na resolução de problemas, na contextualização, na capacidade investigativa e no desenvolvimento de competência crítica para utilizar a FE em diversos contextos e, diante de fatos e dados confiáveis, poder tomar decisões conscientes em prol de si mesmo e de sua comunidade. Todas essas habilidades vão ao encontro das competências específicas dispostas em Brasil (2018), que devem ser desenvolvidas pelos estudantes de EM ao estudar Matemática.

Deste modo, faz-se necessário analisar as pesquisas sobre o ensino e aprendizagem da $\mathrm{FE}$ na $\mathrm{EB}$, identificando quais aspectos da $\mathrm{BNCC}$ já foram considerados e quais as lacunas que ainda persistem e precisam ser investigadas, tendo como objetivo dar subsídios teóricos e empíricos de qualidade, principalmente para os professores, para que o ensino dessa função possa ser aprimorado, de modo a desenvolver as habilidades elencadas pela BNCC.

\section{Percurso metodológico}

Esta pesquisa é de natureza exploratória quanto aos objetivos e bibliográfica quanto à coleta de dados (FIORENTINI; LORENZATO, 2012). A busca por teses e dissertações sobre o ensino e aprendizagem da FE no EM foi realizada no Banco de Teses e Dissertações da Coordenação de Aperfeiçoamento de Pessoal de Nível Superior (CAPES).

O descritor de busca utilizado foi "FE", fornecendo 184 resultados. Utilizou-se, ainda, o Thesaurus Brasileiro da Educação (Brased), mas não foram encontrados outros descritores que pudessem nortear a busca. Foram utilizados alguns filtros disponíveis na plataforma para refinar a busca, de modo a selecionar as produções na área de Educação e Ensino de Matemática, sendo encontrados 84 resultados. Para fins de análise do material selecionado, 
utilizou-se a Análise de Conteúdo, pois esta possui uma função heurística que se caracteriza pela busca exploratória e enriquecimento da possibilidade de descobertas.

Bardin (2016, p. 125) afirma que a análise de conteúdo é composta por três etapas: “1) pré-análise; 2) exploração do material; e 3) tratamento dos resultados, inferência e interpretação.”. Na pré-análise, foi realizada a leitura flutuante pelo título e resumo dos 84 trabalhos, sendo excluídos aqueles que não tratavam sobre o ensino e aprendizagem da FE no EM, assim como os que não apresentavam desenvolvimento de propostas de ensino, resultando em 51 trabalhos, sendo 50 dissertações e uma tese.

$\mathrm{Na}$ segunda fase, para criar as categorias de análise, a priori, das competências e habilidades da BNCC, foi realizada uma leitura flutuante em um recorte da BNCC, incluindo as cinco competências específicas para a Matemática no EM (BRASIL, 2018, p. 531) e as habilidades referentes às funções exponenciais (Quadro 1).

A seguir, foi aplicada uma análise estatística utilizando o programa "Estatística das letras, palavras e períodos" (BORTOLOSI, 2013), que identificou 459 palavras, sendo que, destas, 226 eram diferentes entre si. Os dados foram exportados para uma planilha eletrônica e foram excluídas as palavras que não apresentavam nenhum grau de significado para a análise proposta, como artigos, preposições etc., assim como as palavras "matemática (o)(s)", "exponencial(is)" e "funções", por remeterem diretamente ao objeto de investigação.

Foi realizado o agrupamento das palavras que, segundo o contexto do texto, deveriam figurar como compostas. Após o tratamento, resultaram 33 agrupamentos de palavras e um total de 175 ocorrências que originaram as nove categorias de análise, sistematizadas no Quadro 2, as quais, juntas, somam $69 \%$ da frequência de palavras, na análise estatística.

Em seguida, as pesquisas foram lidas na íntegra, identificando-se as unidades de registro presentes em cada uma delas, conforme categorias do Quadro 2. Para cada categoria de análise, foram registradas, em planilha eletrônica, além da ocorrência ou não em cada uma das pesquisas, mas também como tal categoria estava presente, quais as palavras mais frequentes, o enfoque e a forma de utilização pelos pesquisadores. Ressalta-se que não foram consideradas as categorias como mutuamente excludentes, mas sim a possibilidade de múltiplas ocorrências de delas nas diversas pesquisas em combinado ou separadamente.

Quadro 2 - Categorias de análise com foco na BNCC 


\begin{tabular}{|c|c|}
\hline $\begin{array}{l}\text { Definições, conceitos e } \\
\text { propriedades }\end{array}$ & Caracterização da função, definição, propriedades, demonstrações etc. \\
\hline os de & $\begin{array}{l}\text { Uso de, no mínimo, dois registros de representação como o gráfico, tabelas, álgebra, } \\
\text { língua materna ou outros que permitam representar o objeto matemático com a } \\
\text { presença de tratamento e conversão desses registros. }\end{array}$ \\
\hline Uso de $t$ & Uso de recursos como calculadoras, softwares, aplicativos etc. \\
\hline Intradisciplin & $\begin{array}{l}\text { Relação com outros conteúdos matemáticos como funções logarítmicas, progressões } \\
\text { geométricas, juros compostos, estatística etc. }\end{array}$ \\
\hline tica & Relação com conhecimentos como economia, inflação, juros, orçamento familiar etc. \\
\hline $\begin{array}{l}\text { Contextualização: } \\
\text { outros contextos }\end{array}$ & $\begin{array}{l}\text { Utilização de contextos (reais ou fictícios) para a aprendizagem dos estudantes, numa } \\
\text { perspectiva de uma matemática que dê sentido para a função exponencial para além } \\
\text { dos números em contextos que sejam, de fato, significativos para a realidade social } \\
\text { dos estudantes em questão. }\end{array}$ \\
\hline Interdisciplinaridade & $\begin{array}{l}\text { Relação com outras áreas de conhecimento como ciências humanas, ciências sociais } \\
\text { e ciências da natureza. }\end{array}$ \\
\hline $\begin{array}{l}\text { Habilidades de } \\
\text { comunicação }\end{array}$ & $\begin{array}{l}\text { Propiciação ao desenvolvimento da comunicação em suas diversas modalidades, da } \\
\text { argumentação crítica e do uso de diversas linguagens. }\end{array}$ \\
\hline
\end{tabular}

Fonte: elaborada pelos autores

Observa-se que, dentro da contextualização, foram criadas duas categorias: uma, exclusiva para a Matemática Financeira; e a outra, englobando outros contextos. Neste processo, uma dissertação foi excluída nesta etapa pois, embora citasse em seu resumo, no corpo do texto não tratava do ensino da FE, restando 50 trabalhos, que foram agrupados conforme o Quadro 3.

Quadro 3 - Trabalhos analisados nas categorias criadas com foco na BNCC

\begin{tabular}{|c|c|c|}
\hline & Autores & Quantidade \\
\hline 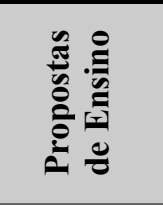 & $\begin{array}{l}\text { Barra (2017), Borges (2014), Coelho (2016), Dantas (2014), Einhardt (2016), } \\
\text { Ferri (2014), Lima (2014), Lima (2016), Maia (2017), Martinez (2015), Moura } \\
\text { (2018), Oliveira (2013), Oliveira (2014), Piano (2016), Ramos (2015), Robalo } \\
\text { (2014), Santos (2014), Santos (2015), Sena (2014), Silva, C. (2015), Silva, R. } \\
\text { (2015), Silva (2016), Tavoni (2013), Villani (2017). }\end{array}$ & 24 \\
\hline 象 & $\begin{array}{l}\text { Angelucci (2014), Angiolin (2009), Bezerra (2014), Bonotto (2015), Borges } \\
\text { (2013), Braz (2007), Brucki (2011), Cruz (2018), Fonzar (2014), Gadioli (2015), } \\
\text { Gomes (2017), Macalós (2019), Marchetto (2017), Mendes (2017), Mendonça } \\
\text { (2017), Menezes (2018), Narcizo (2016), Olgin (2011), Oliveira (2018), Prestes } \\
\text { (2019), Rozanski (2015), Santos (2018), Silva (2014), Sousa (2016), Souza } \\
\text { (2010), Toledo (2018). }\end{array}$ & 26 \\
\hline & Total & 50 \\
\hline
\end{tabular}

Fonte: dados da pesquisa

Nas seções a seguir são apresentados os resultados da análise dos trabalhos agrupados, segundo as categorias elencadas no Quadro 2, observando-se que um trabalho pode figurar em mais de uma categoria, as quais não são mutuamente exclusivas e, além disso, em cada pesquisa estiveram presentes diversos aspectos que possibilitaram analisá-las, à luz das diversas categorias neste lócus de análise. 
Esta categoria foi identificada em 21 dos 50 trabalhos analisados. Dentre estes, Oliveira (2013), Martinez (2015), Toledo (2018), Macalós (2019), Angiolin (2009), Coelho (2016), Ramos (2015) e Silva C. (2015), que consideram a resolução de problemas como abordagem para o ensino da FE; entretanto, apenas os quatro primeiros autores apresentaram fundamentação teórica para tal. Em alguns trabalhos existe uma indefiniç̧ão quanto à resolução de problemas, apresentando, inclusive, diversas terminologias, como problemas, exercícios, atividades e tarefas para descrever um mesmo objeto.

A pesquisa de Oliveira (2013) se destaca por conseguir aliar a abordagem da resolução de problemas com a modelagem matemática, mostrando, assim, uma possibilidade interessante para o ensino das funções exponenciais. Na modelagem Matemática, destacam-se os trabalhos de Ferri (2014), que propõe um processo de experimentação para que os estudantes possam produzir dados e modelar os fenômenos de comportamento exponencial; o de Tavoni (2013), que apresenta uma proposta de ensino com a coleta de dados reais; e o de Maia (2017), que propõe atividades contextualizadas em que os estudantes constroem os conceitos e aprendizagens por meio da modelagem matemática. Além destes, Brucki (2011) e Narcizo (2016) também desenvolvem suas pesquisas utilizando a modelagem matemática.

A resolução de problemas e a modelagem matemática se configuram como lugar de destaque nas pesquisas analisadas, aproximando-se do que sugere a $\mathrm{BNCC}$ ao afirmar que o estudante deve desenvolver habilidades para interpretar, analisar e resolver problemas. Outras abordagens também foram verificadas, como aulas de caráter investigativo nos trabalhos de Bezerra (2014), Bonotto (2015), Sousa (2016), Mendes (2017) e Prestes (2019), além da utilização de materiais concretos para simulação e produção de dados na pesquisa de Braz (2007), o ensino por atividades em Silva (2014) e as situações didáticas em Rozanski (2015).

Verifica-se que, embora haja um amplo repertório de tendências da Educação Matemática nessas pesquisas, são poucos os trabalhos em que os estudantes têm a oportunidade de trabalhar com situações reais e com a coleta de dados, pois a maioria deles utilizou dados já produzidos e, em muitos casos, fictícios. Deste modo, é necessário investir em pesquisas em que o estudante tenha um papel ativo, conforme propõe a BNCC.

\section{Definições, conceitos e propriedades}

Termos como definições, propriedades, conceitos, características e demonstrações foram verificados na maioria das pesquisas analisadas, sendo que apenas dez deles não tinham uma preocupação explícita e central sobre esses aspectos. Em alguns trabalhos, principalmente os produzidos nos mestrados profissionais, dedicam-se um ou mais capítulos exclusivamente 
para explorar conceitos, definições e demonstrações da FE, em que se destaca uma preocupação excessiva com o caráter algébrico da função.

Tais evidências nos permite refletir que, embora haja uma preocupação em promover um ensino da FE de forma mais articulada com as tendências em Educação Matemática, como a modelagem matemática e a resolução de problemas, ainda existe uma forte herança do ensino pautado no paradigma do exercício e a super valorização de fórmulas e demonstrações, o que corrobora com o que já foi citado anteriormente acerca da necessidade de avanços nos fundamentos teóricos metodológicos, no desenvolvimento dessas pesquisas nesta área.

\section{Registros de representação}

As pesquisas revelaram que no ensino da FE são utilizados diversos registros para mobilizar os conhecimentos e promover a aprendizagem dos estudantes sobre o objeto matemático. O registro algébrico esteve presente em todas as pesquisas, sendo este o principal registro utilizado, em consonância com os achados na categoria "Definições, conceitos e propriedades", que também revelou a predominância da utilização de registros algébricos. Os trabalhos de Olgin (2011), Oliveira (2013), Oliveira (2014) e Dantas (2014), por exemplo, exploraram somente o registro algébrico nas atividades de ensino da FE. Apesar deste registro ser bastante utilizado para explorar as características das funções exponenciais, raramente é utilizado para promover reflexões e interpretações acerca do problema em estudo.

O registro gráfico é o segundo de maior destaque, sendo utilizado em $80 \%$ dos trabalhos. Via de regra, é bastante utilizado para finalizar blocos de atividades, após serem esgotados os exercícios com base no registro algébrico. Percebe-se, ainda, que muitos dos trabalhos utilizam algum tipo de tecnologia no processo de elaboração desses gráficos, dando maior ênfase não ao processo de construção deste, mas sim à compreensão das suas características, revelando um ganho no processo de aprendizagem dos estudantes.

Verificou-se, ainda, que o registro numérico em formato tabular é um dos menos explorados nessas pesquisas pois, em geral, é utilizado somente como um registro intermediário, de passagem, entre o algébrico e o gráfico, sendo dada pouca importância ao seu processo de construção e interpretação. Caso semelhante ocorre com o registro na língua materna, que é utilizado em todas as pesquisas analisadas para enunciar algum problema com situação exponencial, porém, pouco explorado no processo de resolução destes, gerando certo distanciamento entre a linguagem materna e a linguagem matemática.

Nesta categoria se destacam os trabalhos de Souza (2010), Rozanski (2015), Marchetto (2017) e Mendonça (2017), que melhor articulam a utilização de diferentes registros de 
representação para a FE, adotando inclusive a Teoria dos Registros de Representação Semiótica de Raymond Duval como fundamentação teórica, propondo a coordenação entre diferentes registros para possibilitar a aprendizagem do objeto matemático, aproximando-se do que propõe a BNCC ao afirmar que os estudantes precisam saber representar os diversos registros e converter um registro em outro com fluidez.

Fica notória a necessidade de avanços nas pesquisas no que concerne à mobilização dos diversos registros no processo de ensino e aprendizagem da FE pois, embora as pesquisas utilizem, de modo geral, mais de um registro, foi evidenciada uma fragilidade no processo de conversão e coordenação dos registros. É necessário que essa coordenação de registros seja articulada de modo que permita ao estudante visualizar e compreender o mesmo objeto matemático sobre diversas representações, conforme preconiza a BNCC ao afirmar que o estudante deve "Compreender e utilizar, com flexibilidade e fluidez, diferentes registros de representação matemáticos (algébrico, geométrico, estatístico, computacional etc.), na busca de solução e comunicação de resultados de problemas." (BRASIL, 2018, p. 523).

\section{Uso de tecnologias}

O uso das tecnologias foi identificado em 33 dos 50 trabalhos analisados, demonstrando que, assim como é indicado em diversas das habilidades da BNCC para o ensino da FE, a utilização das tecnologias também é uma tendência nas pesquisas analisadas. Os autores utilizaram diversos recursos, 14 no total, conforme o Quadro 4, tendo por objetivo auxiliar o processo de aprendizagem dos estudantes. Os recursos mais utilizados foram o GeoGebra, as calculadoras e as planilhas eletrônicas.

Quanto ao GeoGebra, identificou-se que sua utilização foi prioritariamente destinada a auxiliar os estudantes na compreensão da representação gráfica da função, observação das propriedades e caracteríticas destes gráficos. Nesse sentido, destacam-se os trabalhos de Coelho (2016), Cruz (2018) e Marchetto (2017), que tiveram o foco de suas investigações na utilização deste software para o ensino da FE.

Quadro 4 - As tecnologias na pesquisa em ensino da função exponencial

\begin{tabular}{|c|c|c|}
\hline Tencologia & Autores & Quantidade \\
\hline GeoGebra & $\begin{array}{l}\text { Bonotto (2015), Borges (2014), Coelho (2016), Cruz (2018), Ferri } \\
\text { (2014), Fonzar (2014), Gomes (2017), Macalós (2019), Marchetto } \\
\text { (2017), Martinez (2015), Mendes (2017), Mendonça (2017), Ramos } \\
\text { (2015), Santos (2014), Silva, C. (2015), Villani (2017) }\end{array}$ & 16 \\
\hline Calculadoras & $\begin{array}{l}\text { Barra (2017), Fonzar (2014), Maia (2017), Mendonça (2017), } \\
\text { Menezes (2018), Olgin (2011), Oliveira (2018), Prestes (2019), } \\
\text { Ramos (2015), Santos (2015), Santos (2018), Souza (2010), Villani } \\
(2017)\end{array}$ & 13 \\
\hline
\end{tabular}


Fonte: dados da pesquisa

Os autores destacam benefícios ao utilizarem o GeoGebra, como a facilidade e agilidade que proporciona aos estudantes para confecionar os gráficos, ficando um maior tempo para a interpretação e discussão de suas características, o que é muito mais complexo quando se utiliza papel e lápis, pois os estudantes apresentam dificuldades em calibrar as escalas e em representar a curva exponencial ao ligar os pontos de coordenadas cartesianas.

Ressalta-se que o GeoGebra é uma plataforma de aprendizagem em larga expansão no mundo, devido às possibilidades de recursos disponíveis para se trabalhar diversas áreas do ensino e da aprendizagem da Matemática, tando de forma síncrona como assincrona, bem como online ou offline, e, justamente por isso, a sua adesão em tantas pesquisas.

As calculadoras foram utilizadas ou sugeridas pelos autores para facilitar o processo de cálculos dos estudantes tendo em vista que, ao se trabalhar com a FE, os números aumentam ou decrescem muito rapidamente e, por isso, os cálculos podem se tornar demasiadamente extensos e cansativos. Deste modo, a calculadora proporcionou economia de tempo no processo de cálculo, que pode ser melhor utilizado na compreensão do objeto central de estudo, nas pesquisas e propostas de ensino.

A utilização de planilhas eletrônicas também obteve destaque nestas pesquisas, sendo que o foco de sua utilização era possibilitar ao estudante trabalhar com uma maior quantidade de dados, facilitar os cálculos e gerar gráficos automáticos por meio dos recursos ques estas planilhas disponibilizam. Tal utilização já é indicada na BNCC, em Brasil (2018, p. 526) "Planejar e executar ações envolvendo a criação e a utilização de aplicativos, jogos (digitais ou não), planilhas para o controle de orçamento familiar, simuladores de cálculos de juros compostos, dentre outros, para aplicar conceitos matemáticos e tomar decisões" na habilidade EM13MAT203, reforçando a necessidade da utilização de diversas ferramentas digitais, principalmente nas ações que envolvem o contexto fincanceiro.

Ferri (2014), Coelho (2016) e Maia (2017) propõem sequências didáticas utilizando as planilhas eletrônicas em contextos como aplicação em poupança, juros compostos e o cheque especial, relacionando-os com a FE. Nesta perspectiva, destaca-se o trabalho de Prestes (2019), que utilizou planilhas eletrônicas permitindo aos estudantes simular diversos financiamentos a partir de dados coletados no comério local, verificando a relação exponencial contida nos juros, aproximando-se muito do que é proposto pela BNCC. 
Além dos recursos tecnológicos de maior destaque, diversos outros foram utilizados e, em alguns casos, concomitantemente, como na pesquisa de Gomes (2017), por exemplo, ao utilizar cinco aplicativos distintos no processo de ensino da FE, todos para dispositivos móveis em plataforma android, verificando que estes contribuíram positivamente na motivação e compreensão dos estudantes no estudo da função.

Compreende-se que as pesquisas têm avançado na proposta da utilização das tecnologias digitais no processo de ensino e aprendizagem da FE, tendo assim um diálogo com as habilidades para a Matemática do EM, em Brasil (2018, p. 523), que afirma que os estudantes devem "Investigar e estabelecer conjecturas a respeito de diferentes conceitos e propriedades matemáticas, empregando recursos e estratégias como observação de padrões, experimentações e tecnologias digitais [...]".

\section{Intradisciplinaridade}

Dentre as pesquisas analisadas, 27 relacionaram a FE com um ou mais conteúdos matemáticos, conforme Quadro 5, ficando evidente a possibilidade e a tendência de estabelecer elos e contextos entre os próprios conteúdos matemáticos. A relação entre as progressões, principalmente as geométricas, e a FE recebem destaque nestas pesquisas. Autores como Sena (2014), Sousa (2016) e Marchetto (2017), por exemplo, propõem uma abordagem totalmente integrada entre esses dois conteúdos, ressaltando a possibilidade de serem estudados juntos, embora alguns currículos de EM destinem o ensino das funções exponenciais para a $1^{\mathrm{a}}$ série e o das progressões para a $2^{\mathrm{a}}$ série.

A função logarítmica é o segundo conteúdo que mais foi relacionado à FE nas pesquisas, aparecendo ora de maneira integrada, buscando modelar alguns problemas, ou mesmo fazendo contrapontos entre as características e propriedades de ambas as funções. Entretanto, em alguns trabalhos, como o de Olgin (2011), a relação entre as duas é pouco explorada, sendo quase desconsiderada, embora faça parte da mesma sequência de ensino.

Quadro 5 - Conteúdos matemáticos relacionados às funções exponenciais nas pesquisas

\begin{tabular}{|l|l|c|}
\hline Conteúdos & \multicolumn{1}{c|}{ Autores } & \multicolumn{1}{c|}{ Quantidade } \\
\hline Progressões & $\begin{array}{l}\text { Barra (2017), Bonotto (2015), Borges (2014), Brucki (2011), Ferri } \\
\text { (2014), Fonzar (2014), Marchetto (2017), Moura (2018), Narcizo } \\
\text { (2016), Oliveira (2013), Piano (2016), Ramos (2015), Santos (2015), } \\
\text { Sena (2014), Silva, C. (2015), Silva, R. (2015), Sousa (2016) }\end{array}$ & 17 \\
\hline $\begin{array}{l}\text { Logaritmo, função } \\
\text { logarítmica }\end{array}$ & $\begin{array}{l}\text { Bezerra (2014), Borges (2013), Borges (2014), Fonzar (2014), Olgin } \\
\text { (2011), Piano (2016), Ramos (2015), Silva, R. (2015) }\end{array}$ & 8 \\
\hline Função linear & $\begin{array}{l}\text { Angiolin (2009), Ferri (2014), Fonzar (2014), Moura (2018), Prestes } \\
\text { (2019), Santos (2015), Toledo (2018) }\end{array}$ & 7 \\
\hline Geometria & Angelucci (2014), Bonotto (2015), Mendes (2017) & 3 \\
\hline Contagem & Angel ucci (2014) & 1 \\
\hline
\end{tabular}




\begin{tabular}{|l|l|l|}
\hline Função quadrática & Fonzar (2014) & 1 \\
\hline Trigonometria & Mendes (2017) & 1 \\
\hline
\end{tabular}

Fonte: dados da pesquisa

Esta tendência em estabelecer relações entre as progressões e a FE, e desta com a função logarítmica, é justificada pelo currículo do Mestrado Profissional em Matemática em Rede Nacional (PROFMAT), uma vez que na disciplina "Números e funções reais" tem Lima (2013) como livro texto, que, no Capítulo 8, aborda as relações existentes entre estes tópicos. Tal fato fica ainda mais evidente, pois, das 17 pesquisas que relacionaram as progressões à FE, 15 foram desenvolvidas no PROFMAT, assim como das 8 pesquisas que relacionaram as funções logarítmicas à FE, 6 foram desenvolvidas também neste programa de mestrado. Ainda, das 50 pesquisas analisadas, 34 foram desenvolvidas neste programa de mestrado, mostrado assim a influência do currículo do curso na abordagem das pesquisas.

Esta perspectiva desenvolvida pelas pesquisas também é um dos focos das habilidades elencadas em Brasil (2018, p. 533), na BNCC, que descreve que os estudantes precisam "Identificar e associar sequências numéricas (PG) a funções exponenciais de domínios discretos para análise de propriedades [...]", assim como "Comparar e analisar as representações, em plano cartesiano, das funções exponencial e logarítmica para identificar as características fundamentais [...]" (BRASIL, 2018, p. 531). Isso evidencia que as pesquisas já consideravam diversas dessas perspectivas para o ensino da FE que agora permeiam a BNCC.

\section{Matemática financeira}

A Matemática Financeira também é um conteúdo que recebe destaque nas pesquisas ao ser relacionada com a FE, estando presente em 29 dos trabalhos analisados, conforme Quadro 6. Percebe-se nestas pesquisas uma diversidade de aspectos e temas relacionados, o que vai ao encontro de Brasil (2018, p. 523) ao afirmar na BNCC que, ao estudar Matemática no EM, o estudante deve desenvolver competências para "Utilizar estratégias, conceitos e procedimentos matemáticos para interpretar situações em diversos contextos, sejam atividades cotidianas, sejam fatos das Ciências da Natureza e Humanas, ou ainda questões econômicas”.

Quadro 6 - Temas da Matemática Financeira nas pesquisas sobre função exponencial

\begin{tabular}{|l|l|c|}
\hline Temas & \multicolumn{1}{|c|}{ Autores } & \multicolumn{1}{c|}{ Quantidade } \\
\hline $\begin{array}{l}\text { Aplicação em } \\
\text { poupança }\end{array}$ & $\begin{array}{l}\text { Einhardt (2016), Maia (2017), Villani (2017), Santos (2014), Rozanski } \\
\text { (2015), Prestes (2019), Bezerra (2014), Barra (2017), Bonotto (2015), } \\
\text { Menezes (2018), Ferri (2014), Angiolin (2009) }\end{array}$ & 12 \\
\hline $\begin{array}{l}\text { Investimento } \\
\text { bancário }\end{array}$ & $\begin{array}{l}\text { Lima (2014), Silva, R. (2015), Villani (2017), Prestes (2019), Braz } \\
\text { (2007), Ramos (2015), Coelho (2016) }\end{array}$ & 7 \\
\hline Financiamentos & $\begin{array}{l}\text { Prestes (2019), Braz (2007), Bonotto (2015), Cruz (2018), Angiolin } \\
\text { (2009), Oliveira (2013) }\end{array}$ & 6 \\
\hline
\end{tabular}




\begin{tabular}{|l|l|c|}
\hline Endividamento & Fonzar (2014), Santos (2015), Oliveira (2014), Sousa (2016) & 4 \\
\hline Mercado imobiliário & Macalós (2019), Menezes (2018), Toledo (2018) & 3 \\
\hline Descontos & Silva (2014), Prestes (2019), Sousa (2016) & 3 \\
\hline $\begin{array}{l}\text { Depreciação de } \\
\text { automóveis }\end{array}$ & Rozanski (2015), Marchetto (2017) & 2 \\
\hline Salário & Moura (2018), Prestes (2019) & 2 \\
\hline Empréstimo & Barra (2017), Santos (2015) & 2 \\
\hline
\end{tabular}

Fonte: dados da pesquisa

Barra (2017), por exemplo, teve como objetivo apresentar uma proposta de ensino estabelecendo nitidamente a relação entre esses dois conteúdos. Já Santos (2015) e Prestes (2019), embora tenham como foco central o ensino da Matemática Financeira, também apresentaram relações importantes entre a FE e temas financeiros.

Algumas pesquisas se destacam pela forma como propõem ou conduzem o processo de ensino e aprendizagem e a relação entre esses dois conteúdos matemáticos. Villani (2017), por exemplo, apresenta uma proposta de ensino com temas relevantes, como a reforma previdenciária e, por meio reportagens, propõe diversas discussões para a sala de aula, relacionando a aposentadoria com outros tipos de investimentos complementares, como a poupança e o Certificado Depósito Bancário - CDB. Nesta proposta, além de desenvolver os conteúdos da Matemática Financeira relacionados à FE, os estudantes são conduzidos a refletir sobre questões sociais, economia e planejamento de vida a longo prazo, possibilitando, assim, uma tomada de decisão de forma mais consciente.

Pesquisas como esta se aproximam das competências e habilidades propostas pela BNCC para o ensino e aprendizagem da FE no EM, pois, de acordo com Brasil (2018, p. 526), os estudantes devem desenvolver habilidades para "Planejar e executar ações envolvendo a criação e a utilização de aplicativos, jogos (digitais ou não), planilhas para o controle de orçamento familiar, simuladores de cálculos de juros compostos, dentre outros, para aplicar conceitos matemáticos e tomar decisões".

Outros trabalhos que se destacam são o de Narcizo (2016) e o de Prestes (2019), que relacionam a Matemática Financeira e a FE por meio de atividades contextualizadas em que os próprios estudantes realizam pesquisas de preço e taxas de juros na internet e no comércio local e, a partir dos dados coletados, simulam diversos financiamentos e discutem quais as melhores opções. Nesta abordagem, os estudantes, além de relacionarem a FE às situações cotidianas, são conduzidos a refletir sobre diversos aspectos da Educação Financeira.

Verifica-se que alguns destes trabalhos estabelecem diversos diálogos com a BNCC, investigando práticas educativas que permitam aos estudantes "Interpretar situações econômicas [...] que envolvem a variação de duas grandezas, pela análise dos gráficos das 
funções representadas e das taxas de variação [...]" (BRASIL, 2018, p. 525) e "Resolver e elaborar problemas com funções exponenciais nos quais é necessário compreender e interpretar a variação das grandezas envolvidas, em contextos como o da Matemática Financeira [...]” (BRASIL, 2018, p. 528). Deste modo, percebe-se que, embora muitos trabalhos não tragam a BNCC em sua fundamentação, estabelecem aproximações com ela.

Entretanto, foram identificadas algumas pesquisas que, ao estabelecerem a relação entre a FE e a Matemática Financeira, o fazem de forma descontextualizada, com pouca reflexão acerca dos conceitos e contextos que estão envovidos nas atividades ou tendo como foco apenas os cálculos envolvidos. Em Santos (2014), por exemplo, cria-se uma situação fictícia para a questão envolvendo a Matemática Financeira e a FE e em sua abordagem apenas a resolução dos cálculos algébricos é explorada. Já em Sousa (2016), o contexto das questões, também fictícias, distam muito da realidade, o que não favorece o processo de reflexão dos estudantes sobre os temas estudados.

Diante disto, ainda é necessário avançar na forma como esses elos são realizados, principalmente no que tange aos contextos pois, segundo Brasil (2018), os estudantes precisam desenvolver habilidades de análise e interpretação em diversos contextos que envolvem as questões econômicas e financeiras, verificando o comportamento exponencial e favorecendo a tomada de decisão. Neste sentido, afirma-se que as propostas precisam se aproximar mais dos contextos da vida real e, principalmente, da realidade dos estudantes, para que tais habilidades sejam melhor desenvolvidas.

\section{Contextualização}

Nesta seção são analisadas as demais contextualizações utilizadas nas pesquisas para o ensino das funções exponenciais, conforme Quadro 7, não sendo considerados os já discutidos na seção de Matemática Financeira. Observa-se que alguns autores trabalharam em mais de um contexto.

Quadro 7 - Contextos utilizados nas pesquisas sobre função exponencial

\begin{tabular}{|c|c|c|}
\hline Contextos & Autores & Quantidade \\
\hline $\begin{array}{l}\text { Reprodução de } \\
\text { microrganismos }\end{array}$ & $\begin{array}{l}\text { Silva, R. (2015), Einhardt (2016), Maia (2017), Macalós (2019), Ramos } \\
\text { (2015), Coelho (2016), Barra (2017), Menezes (2018), Marchetto } \\
\text { (2017), Toledo (2018), Cruz (2018), Angiolin (2009), Sousa (2016), } \\
\text { Oliveira (2013), Martinez (2015), Souza (2010), Tavoni (2013) }\end{array}$ & 17 \\
\hline $\begin{array}{l}\text { Decaimento } \\
\text { radioativo }\end{array}$ & $\begin{array}{l}\text { Lima (2014), Einhardt (2016), Maia (2017), Villani (2017), Santos } \\
\text { (2014), Bezerra (2014), Fonzar (2014), Ramos (2015), Menezes (2018), } \\
\text { Oliveira (2014), Sousa (2016), Oliveira (2013), Borges (2014), Brucki } \\
\text { (2011) }\end{array}$ & 14 \\
\hline $\begin{array}{l}\text { Crescimento } \\
\text { populacional }\end{array}$ & $\begin{array}{l}\text { Lima (2014), Maia (2017), Santos (2014), Fonzar (2014), Menezes } \\
\text { (2018), Oliveira (2013), Borges (2014), Souza (2010), Tavoni (2013) }\end{array}$ & 9 \\
\hline
\end{tabular}




\begin{tabular}{|l|l|c|}
\hline $\begin{array}{l}\text { Meia vida de } \\
\text { medicamentos }\end{array}$ & $\begin{array}{l}\text { Einhardt (2016), Maia (2017), Santos (2014), Rozanski (2015), Coelho } \\
\text { (2016), Menezes (2018), Oliveira (2014), Toledo (2018) }\end{array}$ & 8 \\
\hline $\begin{array}{l}\text { Lenda do jogo de } \\
\text { xadrez }\end{array}$ & Ramos (2015), Cruz (2018), Borges (2014), Santos (2018), Silva (2016) & 5 \\
\hline $\begin{array}{l}\text { Lei de resfriamento } \\
\text { de Newton }\end{array}$ & Silva, R. (2015), Villani (2017), Ramos (2015), Borges (2014) & 4 \\
\hline $\begin{array}{l}\text { Descobertas } \\
\text { arqueológicas }\end{array}$ & Silva, R. (2015), Oliveira (2014) & 2 \\
\hline Divisão celular & Einhardt (2016), Bonotto (2015) & 2 \\
\hline Tabagismo & Maia (2017), Bonotto (2015) & 2 \\
\hline Epidemias & Villani (2017), Silva (2014) & 2 \\
\hline $\begin{array}{l}\text { Crescimento de } \\
\text { plantas }\end{array}$ & Silva (2014), Borges (2013) & 2 \\
\hline Redes sociais & Rozanski (2015), Angiolin (2009) \\
\hline
\end{tabular}

Fonte: dados da pesquisa

Os contextos mais utilizados nas pesquisas foram a reprodução de microrganismos, o decaimento radioativo, o crescimento populacional e a meia vida de medicamentos. Tal fato pode ser atribuído por existirem contextos muito semelhantes a estes em Dante (2013), um dos livros de EM mais utilizados no Brasil, sendo que, inclusive, alguns autores afirmaram ter adaptado problemas deste e de outros livros didáticos.

O livro de Lima (2013) também apresenta situações e exercícios para a FE nestes contextos e, como afirmado anteriormente, este é um dos livros textos das disciplinas do mestrado PROFMAT, curso em que foi desenvolvido a maioria das pesquisas analisadas. Deste modo, fica evidenciado que, de modo geral, os autores optaram por contextualizações já conhecidas e utilizadas pela literatura.

Em outras pesquisas, como a de Souza (2010), Sousa (2016) e Gadioli (2015), a contextualização foi utilizada apenas para ilustrar as questões, pois, na proposta ou no desenvolvimento da pesquisa, esses contextos não foram considerados, revelando que o interesse estava centrado nas informações numéricas ali contidas. Diante disso, verificou-se que, em alguns casos, embora as questões e problemas apresentassem potencialidades para explorar a contextualização, esta era desprezada no processo de ensino.

As propostas de ensino de Santos (2014), Martinez (2015) e Barra (2017) também apresentam contextualização de problemas para a FE, entretanto, o direcionamento e discussão dessas propostas têm um foco exclusivamente matemático, analisando propriedades e características da função. Casos semelhantes ocorrem nas pesquisas com estudantes desenvolvidas por Silva (2014), Fonzar (2014) e Marchetto (2017), nas quais a contextualização serviu somente para ilustrar as questões e os problemas, desconsiderando totalmente as possibilidades de discussão dos temas com os estudantes. 
Constata-se que tais pesquisas se distanciam das propostas da BNCC no quesito da contextualização, pois, segundo Brasil (2018, p. 523), os estudantes precisam desenvolver competências para "Articular conhecimentos matemáticos ao propor e/ou participar de ações para investigar desafios do mundo contemporâneo e tomar decisões éticas e socialmente responsáveis". E entende-se que abordagens que desconsideram o contexto relativos aos problemas pouco contribuem para o desenvolvimento de tal competência.

É necessário, segundo Brasil (2018, p. 523), que o estudante desenvolva competência para "Utilizar estratégias, conceitos e procedimentos matemáticos [...] para interpretar, construir modelos e resolver problemas em diversos contextos, analisando a plausibilidade dos resultados e a adequação das soluções propostas [...].”. Sendo assim, é necessário que, além de resolver os problemas, os estudantes possam analisar as soluções encontradas no contexto do problema, verificando se de fato são plausíveis, possíveis e adequados naquele contexto. Diante disto, constata-se que ainda se faz necessário avançar no desenvolvimento de pesquisas que considerem essa perspectiva.

Verificou-se ainda que, em alguns trabalhos como Angelucci (2014), Piano (2016) e Gomes (2017), nenhum contexto foi utilizado, sendo a abordagem totalmente matemática. Da mesma forma, no trabalho de Olgin (2011), embora houvesse o objetivo de contextualizar com a criptografia, não foi verificada relação desta com a FE, sendo apenas utilizada para resolver exercícios onde dado um código e a FE, o estudante deveria apenas decodificar mensagens, aproximando-se muito mais de um exercício mecânico.

Entretanto, outras pesquisas se aproximaram da perspectiva da BNCC na utilização de contextos no ensino da FE, fomentando análise, interpretação e argumentação com base em fatos, dados e contextos relevantes. Tavoni (2013), por exemplo, desenvolve os conceitos da FE com estudantes a partir do contexto do crescimento populacional, com os modelos desenvolvidos por Verhulst e Malthus, propondo discussões sobre estes modelos, analisando semelhanças e diferenças e discutindo com dados reais que deles mais se aproximavam do registro de crescimento de algumas populações.

Maia (2017) também apresenta uma proposta de ensino relacionando a FE a contextos importantes como o decaimento radioativo, utilizando como pano de fundo o famoso acidente com o Césio-137 no Brasil, trazendo informações reais e propondo diversas discussões com os estudantes para que a FE seja trabalhada nesse contexto. Já Macalós (2019) contextualiza por meio do filme "A corrente do Bem" como se daria um crescimento exponencial naquela situação, propondo aos estudantes discussões e estratégias possíveis, questionando sobre a 
possibilidade de a atividade dar certo na vida real, sobre as variáveis envolvidas e sobre as estratégias que poderiam ser utilizadas e suas possíveis falhas.

Assim, fica evidente que as pesquisas em ensino da FE têm tentado utilizar diversos contextos para o ensino deste objeto matemático e que algumas delas apresentam possibilidades bem fundamentadas e articuladas, mas que, de modo geral, a maioria ainda apresenta uma contextualização fraca ou inexistente, que pouco contribui para que o estudante desenvolva habilidades para "Interpretar situações econômicas, sociais e das Ciências da Natureza que envolvem a variação de duas grandezas [...]" (BRASIL, 2018, p. 525), pois desconsideram o contexto, a análise sobre esse contexto e focam apenas nos números que, fora de um contexto, podem não apresentar nenhum significado relevante.

\section{Interdisciplinaridade}

Alguns trabalhos estabeleceram uma relação direta entre a interdisciplinaridade e a contextualização propostas no ensino da FE. Assim como o contexto mais utilizado foi a reprodução de microrganismos, a disciplina de Biologia foi a que mais se estabeleceu relações neste processo de ensino e aprendizagem da Matemática. Tal fato se aproxima das proposições da BNCC, pois, além de indicar a necessidade de estabelecer relações com as diversas ciências, ela dá destaque a relação com a Biologia ao propor, em Brasil (2018, p. 528), que os estudantes devem desenvolver habilidades para "Resolver e elaborar problemas com funções exponenciais [...] em contextos como o da Matemática Financeira e o do crescimento de seres vivos microscópicos, entre outros".

Silva, R. (2015) apresenta uma proposta de ensino na qual, por meio de textos geradores, as questões são contextualizadas e sugere-se discussões acerca destes temas, levando em consideração os conceitos de outras disciplinas, como a Biologia e a Química, para compreender o problema em que será estudada a FE. Mendes (2017), ao desenvolver uma sequência de ensino relacionando a curva catenária à $\mathrm{FE}$, também realiza uma abordagem interdisciplinar ao discutir diversos aspectos da história e arquitetura com os estudantes, incentivando sempre a pesquisa e busca de informações.

Ferri (2014) apresenta uma proposta de ensino por meio da experimentação e modelagem matemática utilizando a lei dos gases. Nesta proposta, o autor destaca diversos aspectos interdisciplinares que podem ser trabalhados em parceria com a disciplina de Química, mostrando assim a potencialidade ao se desenvolver um trabalho interdisciplinar para o ensino da FE. 
Entretanto, nem todos os trabalhos levaram em conta a interdisciplinaridade e, embora alguns deles mostrem potencialidades para tal, acabam por não dialogar com as demais áreas, considerando apenas os aspectos matemáticos. A proposta de Oliveira (2013), por exemplo, mostra um grande potencial de ser trabalhado interdisciplinarmente com a Biologia, a Química e a Geografia, entretanto os direcionamentos do autor revelam que o foco está em discutir apenas os aspectos matemáticos envolvidos nos problemas apresentados.

Diante disto, destaca-se a necessidade de avançar nas concepções e práticas interdisciplinares nas abordagens das pesquisas, de modo a contribuir para que os estudantes desenvolvam competências para "Utilizar estratégias, conceitos e procedimentos matemáticos para interpretar situações em diversos contextos, sejam atividades cotidianas, sejam fatos das Ciências da Natureza e Humanas, ou ainda questões econômicas ou tecnológicas [...]" (BRASIL, 2018, p. 523), contribuindo assim para a formação integral destes sujeitos.

\section{Habilidades de comunicação}

Nesta categoria, identificou-se fragilidade nas pesquisas quanto ao desenvolvimento das habilidades de comunicação com os estudantes. Das 50 pesquisas analisadas, apenas 17 apresentaram propostas ou desenvolvimento de atividades que valorizassem tal habilidade, a saber: Angiolin, 2009; Brucki, 2011; Borges, 2013; Bezerra, 2014; Ferri, 2014; Martinez, 2015; Rozanski, 2015; Bonotto, 2015; Narcizo, 2016; Barra, 2017; Maia, 2017; Mendes, 2017; Cruz, 2018; Oliveira, 2018; Toledo, 2018; Prestes, 2019; e Macalós, 2019.

Destaca-se que foi predominante nestas propostas e pesquisas o trabalho em grupos de estudantes, sendo que, inicialmente, nestes grupos os estudantes deveriam discutir as questões e problemas, propor soluções e efetuar registros para, posteriormente, defender seus achados com os demais grupos da classe e com o professor, argumentando suas soluções principalmente por meio da oralidade.

Martinez (2015), por exemplo, apresenta uma proposta em que, além dos registros escritos das atividades, os estudantes deveriam apresentar e argumentar oralmente tais resoluções, desenvolvendo habilidades de comunicação. Na proposta de Brucki (2011) também é incentivada a argumentação dos estudantes no processo de construção dos conhecimentos, exigindo respostas dissertativas em um contexto, superando o cálculo pelo cálculo. Já na pesquisa desenvolvida por Cruz (2018), além dos registros escritos e da exposição oral, os estudantes também foram solicitados a produzir registros em meio digital, desenvolvendo mais uma possibilidade de comunicar suas ideias. 
Outro fator relevante é que em todas essas pesquisas que se preocuparam com as habilidades de comunicação dos estudantes, os professores assumiram o papel de orientadores, permitindo aos estudantes, em seus próprios grupos, mobilizar conhecimentos para a resolução das atividades propostas, argumentar entre seus pares e entre grupos, ficando o professor como mediador do processo, revelando o protagonismo dos estudantes.

Entretanto, verifica-se que tais pesquisas representam apenas $34 \%$ do universo analisado, demonstrando que as pesquisas ainda precisam evoluir, de modo a promover nos estudantes habilidades para argumentar com base em fatos e dados, utilizando a linguagem matemática e outras formas de linguagem e representações para comunicar informações relevantes e confiáveis, conforme proposto pela BNCC (BRASIL, 2018).

\section{Considerações finais}

Ressalta-se que, das pesquisas analisadas, apenas duas faziam referências à $\mathrm{BNCC}$, fato este justificado pelo documento ter sido homologado em dezembro de 2018. Logo, não era objetivo da maioria das pesquisas analisadas propor ou desenvolver atividades alinhadas a tal documento. Entretanto, os achados nesta pesquisa revelam que, mesmo sobre tais circunstâncias, diversas pesquisas estabelecem diálogos com este documento norteador, revelando aspectos importantes quanto ao uso de diversos registros de representação, ao uso de tecnologias e à relação entre a FE e a Matemática Financeira, evidenciando que muito do que está posto na BNCC já era objeto de investigação nas pesquisas científicas.

Ressalta-se que as tendências da Educação Matemática, como a Resolução de Problemas e a Modelagem Matemática, tiveram destaque nos trabalhos analisados. Tais achados vão ao encontro da BNCC e relevam que estas formas de conduzir o processo de ensino e aprendizagem da Matemática e podem contribuir significativamente para o desenvolvimento de habilidades e competências propostas pela BNCC, revelando ainda uma consonância entre as pesquisas e os documentos norteadores da $\mathrm{EB}$, neste quesito.

Percebe-se, porém, que ainda existem aspectos que necessitam de mais pesquisas e investigações, principalmente aqueles relacionados ao desenvolvimento de contextos significativos, à interdisciplinaridade e ao desenvolvimento de habilidades de comunicação no processo de ensino e aprendizagem da FE, na EB. Destaca-se, ainda, a necessidade do desenvolvimento de pesquisas com mais fundamentações teóricas para o processo de ensino e aprendizagem da Matemática, pois, conforme fora identificado, diversos trabalhos utilizam diversas tendências da Educação Matemática como a modelagem e a resolução de problemas, porém, sem uma fundamentação adequada que subsidie essas pesquisas. 


\section{Referências}

ANGELUCCI, M. Uma abordagem diferente para o ensino da função exponencial no Ensino Médio. 2015. 134 f. Dissertação (Mestrado Profissional em Ensino de ciências exatas) - Universidade Federal de São Carlos, São Carlos, 2015.

ANGIOLIN, A. G. Trajetórias hipotéticas de aprendizagem sobre funções exponenciais. 2009. 196 f. Dissertação (Mestrado em Educação Matemática) - Pontifícia Universidade Católica de São Paulo, São Paulo, 2009.

BARDIN, L. Análise de conteúdo. Tradução de Luís Antero Reto e Augusto Pinheiro. Editora Edições 70. São Paulo, 2016.

BARRA, R. do S. C. Uma proposta de ensino envolvendo os temas juros compostos, função exponencial e progressão geométrica. 2017. 78 f. Dissertação (Mestrado PROFMAT) - Universidade Federal do Pará, Belém, 2017.

BEZERRA, V. F. T. O ensino das funções exponenciais e logarítmicas com a utilização da planilha Excel na forma de aplicativo. 2014. 79 f. Dissertação (Mestrado PROFMAT) Fundação Universidade Federal de Rondônia, Porto Velho, 2014.

BONOTTO, A. K. Ensino e aprendizagem da função exponencial por meio de atividades investigativas e do uso de objeto de aprendizagem. 2015. 125 f. Dissertação (Mestrado Profissionalizante em Ensino de Física e Matemática) - Centro Universitário Franciscano. Santa Maria - RS, 2015.

BONOTTO, A. K.; BISOGNIN, E. Contribuições de um objeto de aprendizagem e dos registros de representações semióticas no Estudo da função exponencial. Novas Tecnologias na Educação. CINTED-UFRGS, v. 13, n. 2, p. 1-11, 2015.

BORGES, U. dos S. Curso de Logaritmo para o Ensino Médio com proposta de atividades alternativas. 2014. 121 f. Dissertação (Mestrado PROFMAT) - Universidade Federal de Juiz de Fora. Juiz de Fora, 2014.

BORGES, W. A. Processos de linguagem na aprendizagem matemática de um grupo de alunos do $1^{\circ}$ ano do Ensino Médio. 2013. 247 f. Tese (Doutorado em Educação Matemática) - Universidade Bandeirante Anhanguera, São Paulo, 2013.

BORTOLOSI, H. J. Estatística das letras, palavras e períodos. 2013. Disponível em: http://www.cdme.im-uff.mat.br/desktop/lpp/lpp-br.html. Acesso em: 12 out. 2020.

BRASIL. Ministério da Educação. Base Nacional Comum da Educação. MEC, 2018.

BRAZ, R. A. F. da S. Uma proposta de utilização de material manipulativo no aprendizado da função exponencial. 2007. 125 f. Dissertação (Mestrado em Ensino das Ciências) - Universidade Federal Rural de Pernambuco, Recife, 2007. 
BREUNIG, R. T.; GABBI, R. Ensino de função exponencial e o jogo de xadrez. In: II Congresso Nacional de Educação Matemática. Anais... Rio Grande do Sul: Projetos Unijui, 2011.

BRUCKI, C. M. O uso de modelagem no ensino de função exponencial. $2011.139 \mathrm{f}$. Dissertação (Profissional em Ensino de Matemática) - Pontifícia Universidade Católica de São Paulo, São Paulo, 2011.

CARDOZO, D.; POSSAMAI, J. P. As Dimensões do Making Sense: a Compreensão de Funções Exponenciais a partir de uma Atividade Investigativa. Acta Scientiae, Canoas, v. 21, n. 4, p. 2-19, Jul./Ago, 2019b.

CARDOZO, D.; POSSAMAI, J. P. Resolver e investigar: possibilidades para o ensino de funções exponenciais. REnCiMa, v. 10 , n. 1, p. 164-183, 2019a.

CARRETA, C. L. A.; GODOY, E. V. O programa nacional do livro didático: um olhar sociocrítico para a abordagem do conceito de função. RPEM, Campo Mourão, v. 7, n. 13, p. 152-180, jan.-jun. 2018.

COELHO, J. R. P. O GeoGebra no ensino das funções exponenciais. $2016.96 \mathrm{f}$.

Dissertação (Mestrado em Matemática) - Universidade Estadual do Norte Fluminense Darcy Ribeiro, Campos dos Goytacazes, 2016.

CRUZ, A. M. da. Potencialidades da utilização do software GeoGebra para o desenvolvimento do conteúdo de funções exponenciais através do smartphone. 2018. 185 f. Dissertação (Mestrado Profissional em Educação Matemática) - Universidade Federal de Ouro Preto, Ouro Preto, 2018.

DANTAS, E. de O. A função exponencial. 2014. 36 f. Dissertação (Mestrado PROFMAT) Universidade Federal Rural de Pernambuco, Recife, 2014.

DANTE, L. R. Matemática: contexto \& aplicações. Editora Ática. $2^{\mathrm{a}}$ ed. São Paulo, 2013.

EINHARDT, I. F. B. Aplicações das funções exponenciais e logarítmicas usando o aplicativo Malmath. 2016. 148 f. Dissertação (Mestrado PROFMAT) - Universidade Federal do Rio Grande, Rio Grande, 2016.

EISERMANN, J. I.; et al. Ensino da função exponencial: Explorando uma abordagem lúdica. In: IV Congresso Internacional de Educação Científica e Tecnológica. Anais... Santo Ângelo: URI, outubro de 2017.

ESPINDOLA, E. Trabalho documental do professor de matemática e projeto de ensino da função exponencial. In: XII Encontro Nacional de Educação Matemática. Anais... São Paulo - SP, 2016.

FERRI, O. E. da S. Progressões e funções: da variação e caracterização das funções do tipo exponencial e logarítmica às técnicas de ajuste de curvas no uso de modelagem matemática. 
2014. 67 f. Dissertação (Mestrado PROFMAT) - Universidade Tecnológica Federal do Paraná, Curitiba, 2014.

FIORENTINI, D.; LORENZATO, S. Investigação em educação matemática: percursos teóricos e metodológicos. $3^{\text {a }}$ edição. Campinas, SP: Editora Autores Associados, 2012.

FONZAR, G. M. B. Crescimento e Decaimento exponencial. 2014. 110 f. Dissertação (Mestrado PROFMAT) - Universidade Federal de Mato Grosso do Sul, Três Lagoas, 2014.

GADIOLI, A. O. Função exponencial: definição, caracterização e aplicações. 2015. 86 f. Dissertação (Mestrado PROFMAT) - Universidade Federal do Espírito Santo, Vitória, 2015.

GOMES, L. A. de F. Aplicativos do sistema operacional Android na aprendizagem de matemática: aplicativos e jogos digitais. 2017. 117 f. Dissertação (Mestrado Ensino de ciências e Educação Matemática) - Universidade Estadual da Paraíba, Campina Grande, 2017.

LIMA, E. L. Números e funções reais. Editora SBM. 1ª edição, Rio de Janeiro. 2013.

LIMA, J. M. Uma Proposta para o Ensino das Funções Exponencial, Seno e Cosseno com o auxílio do Software Winplot. 2017. 73 f. Dissertação (Mestrado PROFMAT) Universidade Federal do Tocantins, Palmas, 2014.

LIMA, M. C. M. de. Função exponencial natural $\mathbf{e}^{\mathrm{x}}$ e número e: uma proposta de abordagem através de aplicações cotidianas e curiosidades. 2016. 95 f. Dissertação (Mestrado PROFMAT) - Universidade Federal do Estado do Rio de Janeiro, Rio de Janeiro, 2016.

LORENCINI, P. B. M.; NOGUEIRA, C. M. I.; REZENDE, V. Registros de representação semiótica, braile e Educação Matemática inclusiva: identificando possibilidades.

Perspectivas da Educação Matemática - INMA/UFMS. v. 11, n. 27, p. 842-862. 2018.

MACALÓS, L. V. Ensino de função exponencial com a metodologia de Resolução de Problemas: Relato de uma prática. 2019. 154 f. Dissertação (Mestrado Profissional em Ensino de Ciências, Matemática e Tecnologias) - Universidade do Estado de Santa Catarina, Joinville, 2019.

MAIA, L. F. M. de Q. Modelação matemática na sala de aula: o conceito de função exponencial numa sequência de atividades para o $1^{\circ}$ ano do Ensino Médio. 2017. $68 \mathrm{f}$. Dissertação (Mestrado PROFMAT) - Universidade Federal de São Carlos, Sorocaba, 2017.

MARCHETTO, R. O uso do software GeoGebra no estudo de progressões aritméticas e geométricas, e sua relação com funções afins e exponenciais. 2017. 148 f. Dissertação (Mestrado em Ensino de Matemática) - Universidade Federal do Rio Grande do Sul, Porto Alegre, 2017.

MARTINEZ, D. A. Função exponencial e seu ensino através da resolução de problemas. 2017. 45 f. Dissertação (Mestrado PROFMAT) - Universidade Estadual Paulista, São José do Rio Preto, 2015. 
MENDES, M. F. A curva catenária como aplicação da função exponencial. 2017.79 f. Dissertação (Mestrado Profissional em Ensino de Ciências Exatas) - Universidade Federal de São Carlos, Sorocaba, 2017.

MENDONÇA, M. S. Registros de representação semiótica, calculadora e o GeoGebra: enlaces possíveis na aprendizagem de função exponencial. 2017. 251 f. Dissertação (Mestrado em Educação Matemática) - Universidade Estadual de Santa Cruz, Ilhéus, 2017.

MENDONÇA, M. S.; PIRES, R. F. Registros de representação semiótica e tecnologias digitais na aprendizagem da função exponencial. In: XII Encontro Nacional de Educação Matemática. Anais... São Paulo: SBEM, 2016.

MENEZES, J. A. F. de. O ensino da função exponencial por meio de atividades. 2018. 247 f. Dissertação (Mestrado Profissional em Ensino de Matemática) - Universidade do Estado do Pará, Belém, 2018.

MOURA, F. E. S. de. A natureza dos Logaritmos e aplicações. 2018. 74 f. Dissertação (Mestrado PROFMAT) - Universidade Federal Rural do Semi-Árido, Mossoró, 2018.

NARCIZO, R. N. V. Investigando a modelagem matemática no ensino de funções afins e exponenciais. 2016. 84 f. Dissertação (Mestrado PROFMAT) - Universidade Federal de Goiás, Catalão, 2016.

OLGIN, C. de A. Currículo no Ensino Médio: uma experiência com o tema Criptografia. 2011. 136 f. Dissertação (Mestrado em Ensino de Ciências e Matemática) - Universidade Luterana do Brasil, Canoas, 2011.

OLIVEIRA, A. J. S. de. O ensino e a aprendizagem de função exponencial em um ambiente de modelagem matemática. 2013. 95 f. Dissertação (Mestrado PROFMAT) Universidade Federal Rural do Semi-Árido. Mossoró-RN, 2013.

OLIVEIRA, M. A. P. de. Sequência didática para o ensino de função exponencial. 2018. 278 f. Dissertação (Mestrado Profissional em Ensino de Matemática) - Universidade do Estado do Pará, Belém, 2018.

OLIVEIRA, M. N. A. de. Análise da contextualização da função exponencial e da função logarítmica nos livros didáticos do ensino médio. 2014. 118 f. Dissertação (Mestrado PROFMAT) - Universidade Federal de Campina Grande. Campina Grande, 2014.

OLIVEIRA, S. G. de.; CALEJON, L. M. C. O jogo torre de Hanói para o ensino de conceitos matemáticos. REnCiMa, Edição Especial: Educação Matemática, v. 7, n. 4, p. 149-158, 2016.

PIANO, C. Diferentes abordagens para o estudo das funções exponenciais e logarítmicas. 2016. 108 f. Dissertação (Mestrado PROFMAT) - Universidade Tecnológica Federal do Paraná, Pato Branco, PR, 2016. 
PRESTES, L. A. da S. Matemática na gestão financeira pessoal. 2019. 66 f. Dissertação (Mestrado PROFMAT) - Universidade Federal do Rio Grande, Rio Grande, 2019.

RAMOS, S. S. A. Logaritmos: uma abordagem didática. 2015. 110 f. Dissertação (Mestrado PROFMAT) - Universidade Federal do Paraná, Curitiba, 2015.

ROBALO, M. S. Aplicação de funções exponenciais e logarítmicas. 2014. 67 f. Dissertação (Mestrado PROFMAT) - Universidade de Brasília, Brasília, 2014.

ROZANSKI, E. F. Metodologia de Ensino do Conceito de Função Exponencial à luz da Teoria das Situações Didáticas. 2015. 116 f. Dissertação (Mestrado PROFMAT) Universidade Tecnológica Federal do Paraná, Pato Branco, 2015.

SANTOS, G. N. Funções exponenciais: uma proposta para professores do ensino médio. 2014. 75 f. Dissertação (Mestrado PROFMAT) - Universidade Federal do Pará, Belém, 2014.

SANTOS, J. dos. Introdução ao conceito da função exponencial: um olhar para a educação inclusiva. 2018. 92 f. Dissertação (Mestrado PROFMAT) - Universidade Tecnológica Federal do Paraná, Curitiba, 2018.

SANTOS, R. O. O ensino da matemática financeira no nível médio e sua importância para a educação financeira do aluno. 2015. 38 f. Dissertação (Mestrado PROFMAT) Universidade Federal da Bahia, Salvador, 2015.

SENA, A. S. Progressões geométricas integrada à função exponencial: uma abordagem ao Ensino Médio. 2014. 71 f. Dissertação (Mestrado PROFMAT) - Universidade Federal do Pará, Belém, 2014.

SILVA, A. L. da; PANOSSIAN, M. L. Situações de ensino da função exponencial nos livros didáticos. In: VII Congresso Internacional de Ensino da Matemática. Anais... Canoas: ULBRA, 2017.

SILVA, C. A. da. A torre de Hanói como ferramenta facilitadora do processo de ensino aprendizagem de função exponencial e resolução de problemas. 2017. 62 f. Dissertação (Mestrado PROFMAT) - Universidade Federal Rural do Semi-Árido, Mossoró, 2015.

SILVA, F. K. da.; COSTA, C. P. da. O ensino de funções exponenciais por engrenagens robóticas. Revista Texto livre Linguagem e Tecnologia. v. 13, n. 2, p. 238-270, mai.-ago., 2020 .

SILVA, R. F. da. Função exponencial e logarítmica. 2016. 118 f. Dissertação (Mestrado PROFMAT) - Universidade Estadual Paulista “Júlio de Mesquita Filho”, São José do Rio Preto, 2016.

SILVA, R. J. A. Contexto e aplicações das funções exponenciais no ensino médio: uma abordagem interdisciplinar. 2015. 87 f. Dissertação (Mestrado PROFMAT) - Universidade Estadual do Norte Fluminense Darcy Ribeiro, Campos dos Goytacazes, 2015. 
SILVA, S. T. T. da. O ensino das funções exponencial e logarítmica por atividades. 2014. 220 f. Dissertação (Mestrado em Educação) - Universidade do Oeste do Pará, Belém, 2014.

SOUSA, I. R. da S. de. Relação entre função exponencial e progressão geométrica. 2016. 73 f. Dissertação (Mestrado PROFMAT) - Universidade Estadual do Norte Fluminense Darcy Ribeiro, Campos dos Goytacazes, 2016.

SOUZA, C. V. de. A função exponencial no caderno do professor de 2008 da Secretaria do Estado de São Paulo, análise de atividades realizadas por alunos da $2^{\text {a }}$ série do ensino médio. 2010. 173 f. Dissertação (Mestrado em Matemática) - Pontifícia Universidade Católica de São Paulo, São Paulo, 2010.

TAVONI, R. Os modelos de crescimento populacional de Malthus e Verhulst: uma motivação para o ensino de logaritmos e exponenciais. 2013. 70 f. Dissertação (Mestrado PROFMAT) - Universidade Estadual Paulista, Rio Claro, 2013.

TOLEDO, L. A. de. Ensino da função exponencial: análise de resultados. 2018. 124 f. Dissertação (Mestrado PROFMAT) - Universidade Estadual Paulista "Júlio de Mesquita Filho", São José do Rio Preto, 2018.

VILLANI, N. de N. R. O número de Euler no Ensino Médio: propostas de abordagens com aplicações. 2017. 82 f. Dissertação (Mestrado PROFMAT) - Universidade Estadual Paulista, São José do Rio Preto, 2017. 